\title{
Linx
}

Revue des linguistes de l'université Paris X Nanterre

$41 \mid 1999$

L'hypothétique

\section{Condition et conditionnelles en chinois contemporain}

Marie-Claude Paris

\section{OpenEdition}

Journals

Édition électronique

URL : http://journals.openedition.org/linx/791

DOI : 10.4000/linx.791

ISSN : 2118-9692

Éditeur

Presses universitaires de Paris Nanterre

\section{Édition imprimée}

Date de publication : 1 décembre 1999

Pagination : 119-135

ISBN : 0246-8743

ISSN : 0246-8743

Référence électronique

Marie-Claude Paris, «Condition et conditionnelles en chinois contemporain », Linx [En ligne], 41 | 1999, mis en ligne le 27 août 2012, consulté le 02 mai 2019. URL : http://journals.openedition.org/linx/791 ; DOI : 10.4000/linx.791

Ce document a été généré automatiquement le 2 mai 2019.

Département de Sciences du langage, Université Paris Ouest 


\title{
Condition et conditionnelles en chinois contemporain
}

\author{
Marie-Claude Paris
}

\section{Introduction}

1 Les subordonnées conditionnelles en mandarin et, de façon plus générale, les subordonnées dites adverbiales, présentent des caractéristiques syntaxiques qui les opposent clairement à d'autres types de subordonnées, dites nominales. Ces autres subordonnées sont, d'une part, les complétives et, de l'autre, les subordonnées compléments d'un nom.

2 Les complétives figurent à droite du verbe matriciel et ne contiennent aucune marque de subordination. C'est pourquoi les positions initiale et finale de la subordonnée, notée P, sont marquées par zéro dans l'exemple (1):

\begin{tabular}{|l|l|}
\hline$(1)$ & wo xiang P [ $\varnothing$ ta jintian bu hui lai $\varnothing]$ \\
\hline & je penser [ il aujourd'hui Neg'. pouvoir venir] \\
\hline
\end{tabular}

Je crois qu'il ne viendra pas aujourd'hui.

3 Les subordonnées compléments du nom sont marquées par un relateur nominal de, qui figure en position finale de la subordonnée, cf. (2). La subordonnée précède la base nominale yuanyin 'raison'.

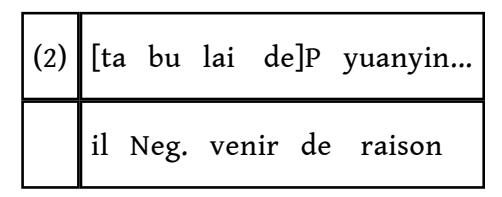

La raison pour laquelle il ne veut pas venir... 
4 Les énoncés complexes constitués de propositions adverbiales et de principales présentent deux caractéristiques distributionnelles frappantes :

5 a) ils contiennent une construction corrélative ;

6 b) les subordonnées précèdent, de façon très préférentielle, les principales.

7 Les constructions corrélatives sont des constructions discontinues dont l'un des membres figure dans la subordonnée et l'autre dans la principale. On appelle conjonction le premier élément et corrélateur le second. Dans (3) ruguo 'si' est la conjonction et jiu le corrélateur. On note $\mathrm{P}$ la subordonnée et $\mathrm{Q}$ la principale, respectivement.

\begin{tabular}{|l|ll|}
\hline (3) & {$[$ ruguo xia da yu] P, [ta jiu bu } \\
\hline \hline & si descendre grand pluie il jiu Neg. \\
\hline
\end{tabular}

S'il pleut fort, il ne viendra pas.

hui lai] $\mathrm{Q}$

pouvoir venir

8 Le premier terme de la construction corrélative peut être une conjonction, comme ruguo 'si' dans (3) ; ce peut aussi être une base nominale, fonctionnant comme la tête de la subordonnée, sur le modèle des compléments du nom présentés en (2) ci-dessus. Dans ce cas, le marqueur nominal de, qui est le relateur par excellence des constructions nominales figure en fin de subordonnée, comme l'illustre (4). Le nom base de cette construction complément du nom est hua 'parole'.

\begin{tabular}{|l|l|}
\hline (4) & [(wo mingtian you kong de) hua $]$ P, [jiu daying \\
\hline & je demain avoir vide de parole jiu promettre \\
\hline
\end{tabular}

Si demain j'ai le temps, je te promets de t'accompagner au cinéma.

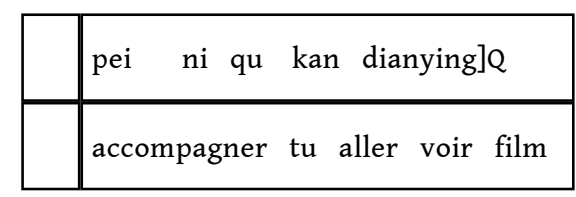

9 La combinaison de marques 'conjonction + base nominale + corrélateur' est aussi attestée, cf. (5), tout comme l'est l'absence totale de marques dans (6).

\begin{tabular}{|l|ll|}
\hline (5) & {$[($ wo ruguo mingtian you kong de $)$ hua $] \mathrm{P}, \quad[j i u$} \\
\hline \hline & je si demain avoir vide de parole jiu \\
\hline
\end{tabular}


Si demain j'ai le temps, je te promets de t'accompagner au cinéma.

daying pei ni qu kan dianying]Q

promettre accompagner tu aller voir film

10 La construction corrélative ne figure pas dans (6): on a affaire à une suite paratactique, dans laquelle la seule co-présence des propositions permet d'inférer le lien conditionnel.

(6) ni bu lai, wo bu zou

tu Neg. venir je Neg. Partir

Si tu ne viens pas, je ne partirai pas.

11 Après cette présentation très rapide de quelques caractéristiques des adverbiales conditionnelles en mandarin, je vais m'attacher à décrire de façon plus précise certains marqueurs entrant dans les constructions corrélatives.

\section{Les marqueurs en corrélation dans les énoncés complexes et leurs valeurs sémantiques}

12 Les marqueurs en corrélation sont listés dans les grammaires du mandarin ${ }^{2}$ sans que soit établie de relation sémantique entre le subordonnant et le corrélateur qui entre en construction avec lui. Ainsi trouve-t-on souvent des paradigmes tels que :

\begin{tabular}{|l|l|l|}
\hline relation sémantique & subordonnants & corrélateurs \\
\hline conditonnelles & $\begin{array}{l}\text { ru(guo), yao(shi), jiashi } \\
\text { 'si' }\end{array}$ & jiu, bian, name \\
\hline causales & $\begin{array}{l}\text { yin, yinwei, yiner } \\
\text { 'parce que' } \\
\text { ji, jiran, jibian } \\
\text { 'puisque' }\end{array}$ & $\begin{array}{l}\text { suoyi } \\
\text { jiu, na(me) }\end{array}$ \\
\hline concessives & $\begin{array}{l}\text { buguan, bulun, wulun } \\
\text { 'n'importe' } \\
\text { suiran, jinguan } \\
\text { 'quoique' }\end{array}$ & ye, dou \\
dan(shi)
\end{tabular}




\begin{tabular}{|l|l|l|}
\hline temporelles & $\begin{array}{l}\text { de shihou, yihou, yiqian } \\
\text { 'quand, après, avant' }\end{array}$ & jiu \\
\hline
\end{tabular}

13 Il n'est pas établi, non plus, de relation entre la forme propre du subordonnant et d'autres marques formellement identiques dans la langue. Dans les deux paragraphes suivants, je vais très rapidement illustrer la polysémie des conjonctions et des corrélateurs.

\subsection{Les marqueurs de subordination}

14 Une opposition modale forte peut être dressée entre les subordonnants relevant de la modalité du 'certain' et ceux relevant du 'non-certain'. Cette opposition s'applique aussi aux corrélateurs.

Les subordonnants des causales 'inférées', qui sont aussi des marqueurs aspectuels, forment un contraste net avec ceux des concessives hypothétiques. Ainsi, par exemple, ji4 ${ }^{3}$, en tant que marqueur verbal indique un accompli ; en tant que conjonction, il marque une cause 'inférée' ('puisque') et peut être suivi de l'anaphorique ran 'c'est ainsi'. Ji2, en tant que marqueur verbal, indique un futur proche ('bientôt') ; en tant que conjonction, il indique une concessive hypothétique 'même si'.

Les subordonnants de condition introduisent des propositions qui ne sont pas des assertions. Leur sémantique est, pour la plupart, transparente. Ruguo 'si' et jiaru 'si' contiennent le morphème ru 'comme', qui marque une comparaison métaphorique. Jiaru 'si' et jiashi 'si' contiennent jia, qui signifie 'faux'. Yao n'est autre que le verbe modal 'vouloir's et yaoshi la concaténation ce verbe avec le verbe shi 'être'. La distribution de yao varie en fonction de son sens. Dans (7) parce qu'il est en tête du groupe verbal et que le quantificateur dou 'tous' a pour champ le pronom sujet tamen 'ils', qui est à sa gauche, yao est interprété comme un verbe modal.

\begin{tabular}{|l|l|}
\hline (7) & tamen dou yao qu faguo \\
\hline & ils tous vouloir aller France \\
\hline
\end{tabular}

Ils veulent tous aller en France.

17 Dans (8a) et (8b) yao précède dou 'tous'. En raison de sa position, il n'est plus la tête du groupe verbal. Comme il précède dou, sa portée est plus large ${ }^{5}$ que celle dou : c'est pourquoi il est interprété comme la conjonction 'si'.

\begin{tabular}{|l|l|}
\hline (8a) & tamen yao dou qu faguo... \\
\hline & ils vouloir/si tous aller France \\
\hline
\end{tabular}

S'ils veulent tous aller en France...

(8b) yao tamen dou qu faguo... 
vouloir/si ils tous aller France

S'ils veulent tous aller en France...

\subsection{Les corrélateurs} principalement binaire. D'un côté, jiu marque une relation de causalité/séquentialité entre deux propositions, c'est pourquoi on le trouve aussi bien dans les principales des temporelles que dans celles des conditionnelles et de certaines causales. De l'autre, le marqueur ye n'est autre que l'adverbe d'adjonction signifiant 'aussi'. Ye est le corrélateur par excellence des concessives hypothétiques et des constructions scalaires: il indique qu'une relation d'implication est niée ${ }^{6}$ ou est contraire à l'attente du locuteur.

19 Les conditionnelles - cf. (3)-(5) ci-dessus - tout comme certaines causales (cf. (9)) et toutes les temporelles (cf. (10)-(11)) entrent en concurrence avec jiu.

\begin{tabular}{|l|l|}
\hline (9) & yinwei you kunnan jiu pai women qu \\
\hline \hline & parce que avoir difficulté jiu envoyer nous aller \\
\hline
\end{tabular}

Ils nous ont envoyés parce qu'il y a/avait des difficultés.

\begin{tabular}{|l|l|}
\hline$(10)$ & ta chi-wan fan yihou jiu zou le \\
\hline & il manger-finir riz après jiu partir P.F. \\
\hline
\end{tabular}

Il est parti après avoir mangé.

\begin{tabular}{|l|l|}
\hline$(11)$ & ta mei lai yiqian, wo jiu zou le \\
\hline \hline & il Neg. venir avant je jiu partir P.F. \\
\hline
\end{tabular}

Je suis parti avant qu'il n'arrive.

20

Jiu n'est pas compatible avec ces différents types de subordonnées, si celles-ci contiennent une restriction. (12)-(14) ci-dessous sont, respectivement, des exemples de conditionnelle, de causale et de temporelle dont le champ est restreint à une occurrence unique : le corrélateur cai - et non jiu - figure alors dans la principale.

\begin{tabular}{|l|l|}
\hline (12) & ruguo Lisi zuo fan de hua, Zhangsan cai (hui) lai \\
\hline & si Lisi faire cuisine de parole Zhangsan cai (pouvoir) venir \\
\hline
\end{tabular}

Zhangsan ne vient/viendra que si Lisi fait la cuisine. 


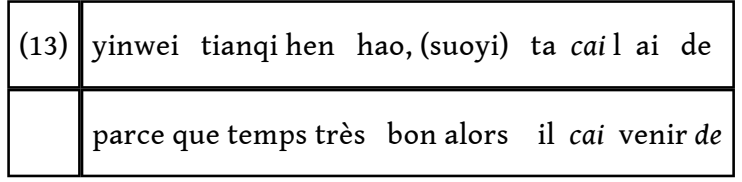

Il n'est venu que parce qu'il fait beau.

\begin{tabular}{|l|l|}
\hline (14) & deng baba hui lai, Zhangsan cai qu \\
\hline \hline & attendre papa rentrer-revenir Zhangsan cai aller \\
\hline
\end{tabular}
qui laisse ouverte la possibilité d'autres énoncés (non dits) : elle entre en cooccurrence avec jiu. Par condition restreinte, j'entends une condition qui est énoncée comme exclusive de toute autre possibilité: elle entre en cooccurrence avec cai. Certains subordonnants comme ruguo, yao, yaoshi 'si' sont non marqués, au sens où ils entrent en corrélation avec l'un ou l'autre de ces deux corrélateurs, cf. (15)-(16).

\begin{tabular}{|l|l|}
\hline (15) & ruguo xia da yu, bisai jiu hui yanqi \\
\hline \hline & si descendre grand pluie match jiu pouvoir retarder \\
\hline
\end{tabular}

S'il pleut beaucoup, le match sera retardé.

\begin{tabular}{|l|l|}
\hline (16) & ruguo xia da yu, bisai cai yanqi \\
\hline & si descendre grand pluie match cai retarder \\
\hline
\end{tabular}



sémantiquement transparents, entretiennent avec l'un ou l'autre marqueur des relations distributionnelles d'exclusion. Wanyi 'au cas où,' qui signifie littéralement 'dix mille-un,' n'est compatible qu'avec jiu, cf. (17)-(18).

\begin{tabular}{|l|l|}
\hline (17) & wanyi you ren zhao Mali, jiu qing ta \\
\hline & si avoir personne chercher Marie jiu prier il \\
\hline
\end{tabular}

Si quelqu'un cherche Marie, demande lui de laisser un message.

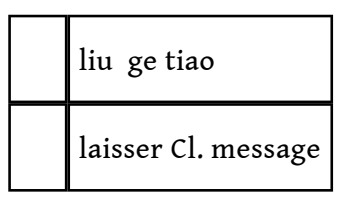

\begin{tabular}{|l|l|}
\hline$(18)$ & *wanyi you ren zhao Mali cai qing ta \\
\hline & $\begin{array}{l}\text { si avoir personne chercher Marie cai prier il } \\
\text { liu ge tiao }\end{array}$ \\
\hline & laisser Cl. message \\
\hline
\end{tabular}

Le subordonnant zhiyao 'seulement-vouloir' = 'si' qui est formé à partir de zhi, opérateur de restriction 'seul(ement)' et du verbe modal yao 'vouloir' indique une condition nonexclusive. Il est compatible avec jiu et non pas avec cai, cf. (19)-(20).

\begin{tabular}{|l|l|}
\hline (19) & zhiyao xuxin, jiu hui jinbu \\
\hline & seulement-vouloir modeste jiu pouvoir progresser \\
\hline
\end{tabular}

Pour peu qu'on soit modeste, on peut faire des progrès.

\begin{tabular}{|l|lr|}
\hline$(20)$ & *zhiyao xuxin, cai hui jinbu \\
\hline & seulement-vouloir modeste cai pouvoir progresser \\
\hline
\end{tabular}

29 Zhiyou, en revanche, qui est constitué de zhi 'seulement' et du verbe you 'avoir' est compatible avec cai, mais ne l'est pas avec jiu, cf. (21)-(22). Une conditionnelle marquée par zhiyou est une conditionnelle exclusive.

\begin{tabular}{|l|ll|}
\hline$(21)$ & ni zhiyou haohao de xuexi, cai hui jinbu \\
\hline
\end{tabular}


tu seulement-avoir bon-bon de étudier cai pouvoir progresser

Tu ne pourras faire des progrès que si tu étudies consciencieusement.

(22) * ni zhiyou haohao-de xuexi, jiu hui jinbu

tu seulement-avoir bon-bon -de étudier jiu pouvoir progresser

Ces deux subordonnants admettent en position finale de subordonnée un nom base qui indique le temps shi 'temps'/'moment', mais interdisent la présence de de hua 'si'. Comparez (23)-(24) à (25)-(26) :

\begin{tabular}{|l|l|}
\hline (23) & *zhiyao tianqi hen leng de hua, Lisi jiu \\
\hline & seulement-vouloir climat très froid de parole Lisi jiu \\
\hline
\end{tabular}

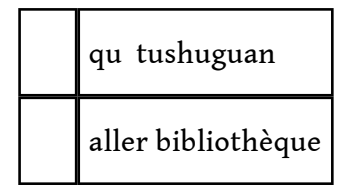

\begin{tabular}{|l|l|}
\hline (24) & zhiyao tianqi hen leng shi, Lisi jiu \\
\hline & seulement-vouloir climat très froid moment Lisi jiu \\
\hline
\end{tabular}

Pour peu qu'il fasse/s'il fait froid, Lisi va à la bibliothèque.

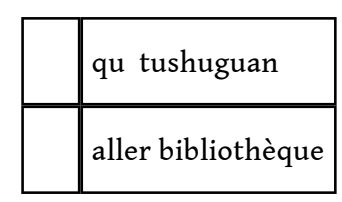

\begin{tabular}{|l|l|}
\hline$(25)$ & *zhiyou tianqi hen leng de hua, Lisi cai \\
\hline & seulement-avoir climat très froid de parole Lisi cai \\
\hline
\end{tabular}

\begin{tabular}{|l|l|}
\hline & qu tushuguan \\
\hline & aller bibliothèque \\
\hline
\end{tabular}

\begin{tabular}{|l|l|}
\hline (26) & zhiyou tianqi hen leng shi, Lisi cai \\
\hline & seulement-avoir climat très froid moment Lisi cai \\
\hline
\end{tabular}


Lisi ne va à la bibliothèque que quand/s'il fait froid.

\begin{tabular}{|l|l|}
\hline & qu tushuguan \\
\hline & aller bibliothèque \\
\hline
\end{tabular}

$31 \mathrm{Au} \mathrm{vu} \mathrm{de} \mathrm{la} \mathrm{cooccurrence} \mathrm{entre} \mathrm{les} \mathrm{subordonnants} \mathrm{et} \mathrm{les} \mathrm{bases} \mathrm{nominales} \mathrm{shi}$ 'temps'/'moment', d'une part, et de hua 'si', d'autre part, on peut établir une distinction entre condition et hypothèse $e^{8}$. On peut poser que les subordonnées marquées par zhiyao... jiu et zhiyou... cai sont des conditionnelles, tandis que les subordonnées marquées par ru (guo), yao(shi), jiaru, jiashi 'si’ sont des hypothétiques. Je ne développerai pas ce point ici.

L'opposition entre les corrélateurs jiu et cai, qui, on vient de le voir, est corrélée à des contraintes distributionnelles claires 9 , permet aussi d'opposer les subordonnées qui sont dans leurs champs du point de vue de leur rôle informationnel.

Comme elles occupent la même place en syntaxe, les subordonnées conditionnelles marquées par jiu et celles marquées par cai ont implicitement été considérées comme jouant un rôle informationnel identique (voir, par exemple, Chao (1968), Tai (1989), Tsao (1990), Tang (1990) et Gasde et Paul (1996)). Pour ces auteurs, toutes les conditionnelles occupe(raie)nt en mandarin la place prototypique de topique. En montrant comment elles se différencient lorsque les énoncés dans lesquels elles figurent sont questionnés, je voudrais prouver que les conditions larges relèvent de l'information ancienne : ce sont des topiques. Les conditionnelles restreintes, en revanche, relèvent de l'information nouvelle et contrastive : ce sont des focus.

\section{Le statut informationnel des conditionnelles}

\subsection{Condition large}

La particule interrogative ma dont le point d'incidence est la fin de l'énoncé permet de questionner un énoncé sans qu'on puisse déterminer directement quelle est la portée de la question, comme l'illustre (27). (28) est la contre partie affirmative de (27).

\begin{tabular}{|l|l|}
\hline (27) & ruguo tianqi hen leng, Lisi jiu hui qu mai shu ma? \\
\hline \hline & si climat très froid Lisi jiu pouvoir aller acheter livre Int. \\
\hline
\end{tabular}

Est-ce que s'il fait froid, Lisi ira acheter des livres?

\begin{tabular}{|l|l|}
\hline (28) & ruguo tianqi hen leng, Lisi jiu hui qu mai shu \\
\hline & si climat très froid Lisi jiu pouvoir aller acheter livre \\
\hline
\end{tabular}

S’il fait froid, Lisi ira acheter des livres. 
Pour former une question, outre la particule finale ma, le mandarin dispose d'une forme marquée sur le verbe, qui se lit de la façon suivante : Verbe + Négation + Verbe. Ainsi, à (29) correspond l'énoncé (30), dans lequel le verbe est questionné.

\begin{tabular}{|l|ll|}
\hline (29) & ni lai $m a ? \quad$ (30) ni lai bu lai $?$ \\
\hline & tu venir Int. $\quad$ tu venir Neg. Venir \\
\hline
\end{tabular}

Est-ce que tu viens? Viens-tu?

La variante grammaticale de (27) dans laquelle le verbe est questionné est non pas (31), mais (32). Dans (31) la question est marquée sur la subordonnée; dans (32) elle est marquée sur la principale. A cette différence syntaxique correspond une différence de portée de la question : dans (32) la principale est dans le champ de la question. C'est donc elle qui porte l'information nouvelle.

\begin{tabular}{|l|l|}
\hline$(31)$ & *ruguo tianqi leng bu leng, Lisi jiu hui qu mai shu? \\
\hline \hline & si climat froid Neg. froid Lisi jiu pouvoir aller acheter livre \\
\hline
\end{tabular}

(32) ruguo tianqi hen leng, Lisi Ø hui bu hui qu mai shu?

si climat très froid Lisi Ø pouvoir Neg. pouvoir aller acheter livre

S'il fait froid, est-ce que Lisi pourra aller acheter des livres?

La distribution du corrélateur jiu dans l'énoncé affirmatif (28) et dans la question (32) est à remarquer. Comme jiu précède nécessairement le verbe modal hui 'pouvoir' dans (28), on s'attend à ce qu'il figure dans (33), qui est la contre partie interrogative de (28). Or (33) est un énoncé agrammatical. Comme l'illustre (32), seule l'absence de jiu, notée $\varnothing$, en position la plus gauche du groupe verbal rend l'énoncé bien formé. Ci-dessous, j'essayerai de proposer une explication du rôle que jiu joue dans les principales.

\begin{tabular}{|l|l|}
\hline (33) & *ruguo tianqi hen leng, Lisi jiu hui bu hui qu mai shu? \\
\hline & si climat très froid Lisi jiu pouvoir Neg. pouvoir aller acheter livre \\
\hline
\end{tabular}

38 Je vais maintenant montrer que les conditionnelles restreintes/nécessaires ne se comportent pas comme les conditionnelles larges lorsqu'elles sont dans le champ de l'interrogation.

\subsection{Condition restreinte}

39 (34) ci-dessous contient, tout comme (27) ci-dessus, la particule interrogative ma. Comme le montre le point d'incidence de l'opérateur de question shi bu shi 'est-ce?' dans (36), 
c'est la subordonnée - et non pas la principale - qui figure dans le champ de la question. (35) est agrammaticale, contrairement à (36).

(34) ni zhiyou caiqu zhei ge banfa cai neng xuehao ma?

tu seul(ement) adopter ce Cl. méthode cai pouvoir étudier-bon Int.

Est-ce que tu ne parviendras à apprendre que si tu adoptes cette méthode?

(35) *ni zhiyou caiqu zhei ge banfa caineng bu neng xuehao?

tu seul(ement) adopter ce Cl. méthode cai pouvoir Neg. pouvoir étudier-bon

\begin{tabular}{|l|l|}
\hline (36) & ni shi bu shi zhiyou caiqu zhei ge banfa cai neng xuehao? \\
\hline & tu être-Neg-être seul(ement) adopter ce Cl. Méthode cai pouvoir étudier-bon \\
\hline
\end{tabular}

Est-ce seulement si tu adoptes cette méthode que tu parviendras à apprendre?

On remarquera, d'une part, que cai reste présent dans la principale de (36), contrairement à jiu qui n'apparaît pas dans (32) et, d'autre part, que l'opérateur de question est shi bu shi 'est-ce?' et non pas la question formée sur le verbe de la subordonnée, à savoir caiqu 'adopter'. La présence de shi 'être' est la trace d'un pré-construit ${ }^{10}$.

41 Pour résumer, les exemples (32) et (36) montrent que les conditionnelles larges marquent l'interrogation sur le verbe de la principale, tandis que les conditionnelles restreintes indiquent l'interrogation à l'aide du verbe 'être' précédant le verbe de la subordonnée.

\subsection{Les restrictions de cooccurrence}

42 D'autres restrictions distributionnelles corroborent le fait que les conditionnelles larges et les conditionnelles restreintes ne jouent pas le même rôle au niveau informationnel. Les conditions larges, puisqu'elles ne sont pas des assertions, admettent des indéfinis dans la subordonnée, tels shenme ou renhe 'n'importe' - ce qui n'est pas le cas des conditions restreintes. Comparez (37) et (38):

\begin{tabular}{|l|l|}
\hline (37) & ni yaoshi you shenme/renhe hao zhuyi de \\
\hline & tu si avoir quelconque/quelconque bon idée de \\
\hline
\end{tabular}

Si tu as quelque bonne idée que ce soit, n'oublie pas de m'avertir.

hua, bie wang-le gaosu wo

parole Neg. oublier-Sfx dire je 


\begin{tabular}{|l|l|}
\hline (38) & *ni yaoshi zhidao you shenme/renhe \\
\hline & tu si savoir avoir quelconque/quelconque \\
\hline & yuyanxue de shu cai gaosu wo \\
\hline & linguistique de livre cai dire je \\
\hline
\end{tabular}

Je reviens maintenant sur la distribution de jiu dans les conditionnelles dont la principale est une question.

\subsection{Le rôle joué par jiu}

Jiu occupe la place d'un adverbe. En syntaxe, c'est l'élément le plus à gauche du groupe verbal, sa portée est donc (la plus) large. En sémantique, sa fonction est anaphorique : il reprend à l'intérieur de la principale, la subordonnée $\mathrm{P}$ qui le précède. Jiu indique une dépendance, une relation de cause / consécution qui existe entre $\mathrm{P}$ (la subordonnée) et $\mathrm{Q}$ (la principale). Une telle analyse permet de comprendre trois caractéristiques distributionnelles de jiu.

Premièrement, si l'ordre canonique $\mathrm{P}, \mathrm{Q}$ devient $\mathrm{Q}, \mathrm{P}$, alors jiu ne figure plus dans $\mathrm{Q}$ puisqu'il n'y a plus de relation d'antécédence, et donc d'anaphore, entre $\mathrm{P}$ et $\mathrm{Q}$. La comparaison de (39)-(40) illustre clairement la distribution de jiu.

\begin{tabular}{|l|l|}
\hline (39) & ruguo xia da yu, ta jiu bu hui lai \\
\hline & si descendre grand pluie il jiu Neg. pouvoir venir \\
& S'il pleut fort, il ne viendra pas. \\
\hline
\end{tabular}

\begin{tabular}{|l|l|}
\hline$(40)$ & ta $(* j i u)$ bu hui lai, ruguo xia da yu \\
\hline & il (jiu) Neg. pouvoir venir si descendre grand pluie \\
\hline
\end{tabular}

Il ne viendra pas, s' il pleut fort.

Deuxièmement, jiu n'apparaît pas lorsque la principale n'est pas une assertion. Ainsi dans (32), qui est une question ou dans (37), qui contient un impératif. Dans (32), l'information principale réside dans la question : la protase ne sert que de cadre général de référence à la validité à l'énonciation de la question. Comme la question ne porte pas sur la relation de consécutivité entre $\mathrm{P}$ et $\mathrm{Q}$, la mention de la trace de surface de cette relation, soit jiu, n'a pas lieu d'être.

Troisièmement, dans (41) ci-dessous il en va autrement : jiu apparait, mais, dans ce cas, sa distribution n'est pas celle que l'on attend. D'un côté, jiu n'occupe plus la position la plus à gauche dans le groupe verbal; de l'autre, il entre, de façon préférentielle, mais non obligatoire, en concurrence avec la particule finale ne. Jiu figure sous le verbe modal, et donc se trouve dans la portée de celui-ci, comme l'illustre (41). 


\begin{tabular}{|l|l|}
\hline$(41)$ & ruguo tianqi hen leng, ni hui bu hui jiu chuan qunzi (ne) ? \\
\hline & si climat très froid tu pouvoir Neg. pouvoir jiu porter jupe (Int.) \\
\hline
\end{tabular}

S'il fait/faisait froid, porterais-tu une jupe?

L'énonciation de (41) suppose un pré-construit, par exemple, une association par le locuteur d'une relation de causalité / consécution entre le froid avec le port d'un vêtement long ou chaud. Dans (41) l'énonciateur demande à son interlocuteur si l'association du froid avec le port d'une jupe (relation de causalité/consécution dont il se distance) sera validée. Le marquage en surface de la distanciation du locuteur par rapport à son énonciation est double. D'une part, jiu figure dans le champ du verbe modal hui 'pouvoir', d'autre part, la particule finale ne prend en charge cette distanciation ${ }^{11}$. Comme on a affaire à une question à propos d'une conditionnelle, il est naturel que jiu figure nécessairement en surface, puisque son rôle discursif est d'indiquer la relation entre $\mathrm{P}$ et Q. La traduction par un verbe au conditionnel dans la principale en français essaye de rendre la cooccurrence de jiu et de ne en chinois. Dans (32), contrairement à (41), la protase est comme indépendante de l'acte de question qui la suit.

Je voudrais maintenant décrire une relation de condition marquée au niveau modal. Il s'agit de la contrefactualité.

\section{Les contrefactuelles}

Comme on le sait, en français ou en anglais, la relation de contrefactualité est prise en charge en surface par, d'un côté, les types de temps et la concordance des temps entre protase et apodose et, de l'autre, le cas échéant, par la présence d'une négation, comme le montre le contraste d'interprétation entre (42) et (43).

(42) Si tu ne m'avais pas aidé, je n'aurais pas pu finir à temps.

(43) Si tu ne m'aidais pas, je ne pouvais pas finir à temps.

51 Comme le mandarin ne possède pas de marqueurs correspondant à des temps en français, on s'attend à ce que la contrefactualité soit indiquée non pas par les verbes eux-mêmes mais par des marqueurs dépendant des verbes. Ainsi trouve-t-on, d'un côté, la présence d'anaphore temporelle de référence passée, ou, de l'autre, la présence de la négation. La présence d'un adverbe anaphorique du passé comme dangshi 'à ce moment là'/'alors' rend l'indication d'accompli sur le verbe superflue, comme le prouvent (44) et (45). L'absence du suffixe -le sur le verbe de la subordonnée est grammaticale.

\begin{tabular}{|l|l|}
\hline (44) & ni dangshi yaoshi tichulai-le, ta hui gei ni \\
\hline & tu alors si mentionner-Sfx. il pouvoir donner tu \\
\hline
\end{tabular}


Si tu l'avais mentionné à ce moment là, il aurait pu signer pour toi.

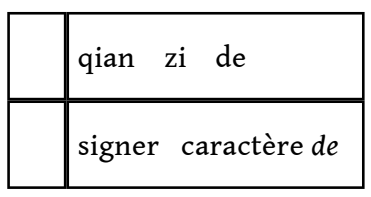

\begin{tabular}{|l|ll}
\hline (45) & ni dangshi yaoshi tichulai- $\varnothing$, ta hui gei ni \\
\hline \hline & tu alors si mentionner- $\varnothing$ il pouvoir donner tu \\
\hline
\end{tabular}

Si tu l'avais mentionné à ce moment là, il aurait pu signer pour toi.

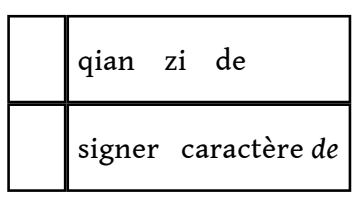

52 Dans (46) la présence de la négation de l'accompli mei indique la contrefactualité.

\begin{tabular}{|l|l|}
\hline (46) & ni yaoshi mei bang wo de mang, wo jiu bu neng \\
\hline \hline & tu si Neg. aider je de pressé je jiu Neg. Pouvoir \\
\hline
\end{tabular}

Si tu ne m'avais pas aidé, je n'aurais pas pu finir ce travail à temps.

\begin{tabular}{|l|l|}
\hline & anshi wancheng zhei jian gongzuo \\
\hline & à temps finir ce Cl. Travail \\
\hline
\end{tabular}

53 Comme l'a montré Eifring $(1988,1995)$, la conjonction yaobushi marque de façon univoque la contrefactualité en chinois contemporain. Yaobushi, formée à partir du verbe modal yao 'vouloir' suivi de la négation (non-accomplie) bu, elle-même suivie du verbe shi 'être'. De façon plus générale, lorsque la négation bu shi 'ne pas être' a un champ large et, donc, figure dans une position plus à gauche que la négation du groupe verbal, on a affaire à une contrefactuelle. (47) et (48) ci-dessous, qui sont empruntés à Eifring (1995), entretiennent une relation de paraphrase. Dans (47), qui est ambigu entre une interprétation hypothétique et une interprétation contrefactuelle, la négation mei you 'ne pas avoir' figure dans le groupe verbal : elle a un champ étroit. Dans (48), qui n'est pas ambigu, la négation bu shi figure à gauche de la proposition subordonnée (tout en étant dans le champ du marqueur d'hypothèse ruguo 'si'): elle a un champ large. L'interprétation ne peut être que contrefactuelle.

\begin{tabular}{|l|l|}
\hline (47) & ruguo Ge Rixin mei you gaosu ta, ta hai bu \\
\hline & si Ge Rixin Neg. avoir dire il il encore Neg. \\
\hline
\end{tabular}


Si Ge Rixin ne lui a pas dit, il ne sait pas encore qu'ils

Si Ge Rixin ne lui avait pas dit, il ne saurait pas encore

zhidao yijing jinru-le xiaoqu

savoir déjà entrer-Sfx. Campus

sont entrés dans le campus.

qu'ils sont entrés dans le campus.

\begin{tabular}{|l|l|}
\hline (48) & ruguo bu shi Ge Rixin gaosu ta, ta hai bu \\
\hline & si Neg. être Ge Rexin dire il il encore Neg. \\
\hline
\end{tabular}

Si Ge Rixin ne lui avait pas dit, il ne saurait pas encore

\begin{tabular}{|l|l|}
\hline & zhidao yijing jinru-le xiaoqu \\
\hline & savoir déjà entrer-Sfx. Campus \\
\hline
\end{tabular}

qu'ils sont entrés dans le campus.

54 Lorsque la principale n'est pas une assertion, la contrefactualité peut être marquée par la négation de l'existence et du passé mei you 'ne pas avoir'. Cette négation, tout comme la négation de l'essence et du futur bu shi, a un champ large et, donc, apparaît dans la position la plus à gauche de la subordonnée, cf. (49).

\begin{tabular}{|l|l|}
\hline$(49)$ & mei you ta gei ni qian, ni bu dei ai e ? \\
\hline & Neg. avoir il donner tu argent tu Neg. devoir subir faim \\
\hline
\end{tabular}

S'il ne t'avait pas donné d'argent, n'aurais-tu pas connu la faim?

Les correspondants des oppositions temporelles (que l'on trouve dans les langues indoeuropéennes) entre hypothétiques et contrefactuelles apparaissent en chinois sous la forme de négations et de particules finales modales différentes. Dans (50)-(51), la particule finale d'assertion $d e^{12}$ s'oppose à le et à $\varnothing$, qui figurent dans (52)-(53). Mei s'oppose à bu dans (50) par rapport (51)-(53). Il faut noter, en passant, que dans une hypothétique le point d'incidence de la négation ne peut être que le groupe verbal, alors que dans une contrefactuelle ce point d'incidence peut être soit le groupe verbal, soit toute la conditionnelle. A point d'incidence différent correspond une négation différente. Mei précède le verbe de la subordonnée dans $(47) /(50)$. Dans $(48) /(51)$ bu shi figure en tête de la subordonnée.

ruguo tamen mei zhao-dao nei tiao hu, shi bu

si ils Neg. chercher-arriver ce Cl. lac être Neg. 
S'ils n'avaient pas trouvé ce lac, ils n'auraient pas survécu.

hui huo-xialai de

pouvoir vivre-continuer de

(51) yaobushi tamen zhao-dao nei tiao hu le, shi

si ils chercher-arriver ce Cl. lac P.F. être

S'ils n'avaient pas trouvé ce lac, ils n'auraient pas survécu.

bu hui huo-xialai de

Neg. pouvoir vivre-continuer de

\begin{tabular}{|l||l|}
\hline (52) & ruguo tamen zhao-bu-dao nei tiao hu, jiu \\
\hline & si ils chercher-Neg.-arriver ce Cl. lac jiu \\
\hline
\end{tabular}

S'ils ne trouvent pas ce lac, ils ne survivront pas.

\begin{tabular}{|l|l|}
\hline & bu hui huo-xialai $\varnothing$ \\
\hline & Neg. pouvoir vivre-continuer $\varnothing$ \\
\hline
\end{tabular}

(53) ruguo tamen zhao-bu-dao nei tiao hu, jiu

si ils chercher-Neg.-arriver ce Cl. lac jiu

S'ils ne trouvaient pas ce lac, ils ne survivraient pas.

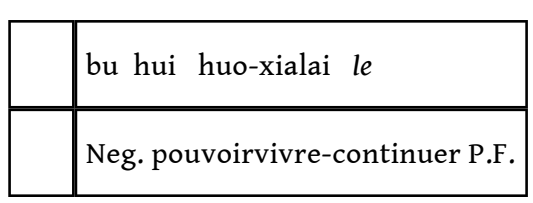

Dans (50)-(51) les subordonnées prennent en charge l'opposition sémantique entre hypothèse et contrefactuelle. Dans (52)-(53), les subordonnées sont identiques, mais l'absence ou la présence de particule finale dans les principales équivalent à l'indication de repères temporels différents en français. 


\section{Conclusion}

Dans cette recherche j'ai essayé d'esquisser quelques grandes oppositions qui, en chinois contemporain, traversent les relations de subordination en général et, plus particulièrement les relations de condition. Il s'agit des oppositions entre :

- condition et hypothèse,

- condition large et condition restreinte,

- condition nécessaire et condition suffisante,

- conditionnelles en jiu par rapport à celles en cai du point de vue modal et informationnel,

- condition et contrefactualité.

\section{BIBLIOGRAPHIE}

CHAO, Y. (1968), A Grammar of spoken Chinese, University of California Press, Berkeley.

COYAUD, M. (1969), « Les phrases hypothétiques en chinois contemporain. Problèmes de méthode », Word, 25, pp. 87-103.

EIFING, H. (1988), « The Chinese counterfactual », Journal of Chinese Linguistics, 16, pp. 193-218.

EIFING, H. (1995), Clause Combination in Chinese, Leiden, Brill.

GASDE, H.-D. et PAUL, W. (1996), « Functional categories, topic prominence, and complex sentences in Mandarin Chinese », Linguistics, 34, pp. 263-294.

HAIMAN, J. (1974), « Concessives, conditionals and verbs of volition », Foundations of Language, 11, pp. 342-360.

HUANG, Sh. (1981), « On the scope phenomena of Chinese quantifiers », Journal of Chinese Linguistics, 9.2, pp. 226-243.

MA, Z. (1994), Petite grammaire pratique du chinois. [Jianming shiyong hanyu yufa], B. Niederer, trad., Paris, Peeters.

PARIS, M.-C. (1979), Nominalization in Mandarin Chinese. The Morpheme de and the shi... de Construction, Paris 7 University, Département de Recherches Linguistiques.

PARIS, M.C. (1983), « Marqueurs et corrélateurs hypothétiques en mandarin », Verbum, VI. 3, pp. 327-342.

PARIS, M.-C. et TAMBA, I. (1984), « Quelques aspects de la concession en chinois et en japonais », in P. VALENTIN (ed), Linguistica Palatina Colloquia I, Paris, Presses de l'Université Paris-Sorbonne, pp. 163-180.

TAI, J. H.-Y. (1989), « Toward a cognition-based functional grammar of Chinese », in J. TAI et F. HSUEH (eds), Functionalism and Chinese Grammar, Seton Hall, Monograph Series, Journal of the Chinese Language Teachers' Association, pp. 187-226. 
TANG, J. (1990), Chinese Phrase Structure and the extended X' Theory, Cornell University, Thèse de Ph. D.

TENG, S. (1974), « Negation in Chinese », Journal of Chinese Linguistics, 2, pp. 125-140.

TSAO, F. (1990), Sentence and Clause Structure in Chinese: A Functional Perspective, Taipei, Student Book Co.

\section{NOTES}

1. Les abréviations utilisées sont les suivantes : Cl. : Classificateur ; Int. : Interrogation ; Neg. : Négation ; P.F. : Particule Finale ; Sfx : Suffixe.

2. Voir, par exemple, Ma, 1994.

3. Les chiffres 2 et 4 après ji renvoient au deuxième et au quatrième tons, respectivement.

4. Voir Haiman, 1974.

5. Pour plus de détails sur la relation entre ordre des quantificateurs et leur portée, voir Huang (1981). Un autre test distributionnel permet d'opposer (7) et (8a)-(8b). Lorsque yao fonctionne comme verbe modal, il ne commute pas avec yaoshi 'si'. Dans (8a)-(8b), en revanche, yao et yaoshi alternent.

6. Pour plus de détails, voir Paris et Tamba, 1984.

7. L'opposition 'large' / 'restreint' me semble mieux décrire les relations marquées par les corrélateurs jiu et cai que l'opposition 'suffisante'/nécessaire', proposée dans Paris, 1983. Les conjonctions zhiyao/zhiyou relèvent de cette dernière opposition.

8. Coyaud (1969) n'opère pas cette distinction.

9. Les subordonnées en corrélation avec jiu admettent d'être coordonnées à l'aide d'une coordination de propositions, c'est-à-dire une coordination externe, cf. (i) :

(i) ruguo xia yu erqie/bingqie ruguo wo de pengyou

si descendre pluie et/et si je de ami

S'il pleut et si mon ami vient, je ne te téléphonerai pas.

lai, wo jiu bu gei ni da dianhua

venir je jiu Neg. donner tu battre téléphone

Les subordonnées en corrélation avec cai interdisent, par définition, la coordination externe, puisqu'elles indiquent une condition unique. Elles n'admettent qu'une coordination interne, c'est-à-dire au niveau des constituants de la proposition, par exemple, le groupe verbal. Comparez (ii) et (iii).

(ii): * zhiyou Mali gei wo yi kuai tang bingqie seu(lement) Marie donner je un $\mathrm{Cl}$. bonbon et

*Seulement si Marie me donne un bonbon et seulement.

zhiyou Lisi qinqin wo, wo cai zou

seul(ement) Lisi embrasser je je cai partir

si Lisi m'embrasse, je partirai

zhiyou Mali gei wo yi kuai tang bingqie seul(ement) Marie donner je un $\mathrm{Cl}$. bonbon et Je ne partirai que si Marie me donne un bonbon et m'embrasse. qinqin wo, wo cai zou

embrasser je je cai partir

Dans (ii), bingqie 'et' coordonne des propositions ; dans (iii) bingqie 'et' coordonne des groupes verbaux.

10. Pour plus de détails sur le rôle de shi, voir Teng, 1974.

11. Dans Paris (1981: 416), je considère que « ne oriente l'énoncé vers sa dénégation possible ». 
12. Cette particule de n'est autre que le marqueur de nominalisation attesté dans (2) ci-dessus, cf. Paris, 1979.

\section{RÉSUMÉS}

Les subordonnées conditionnelles sont étudiées tout d'abord du point de vue distributionnel, puis du point de vue informationnel. Sont présentées, d'une part, la polysémie des subordonnants, de l'autre, les corrélations entre subordonnants et corrélateurs et, enfin, certaines restrictions de cooccurrences entre les subordonnants et les noms bases des subordonnées. A l'opposition entre les corrélateurs jiu et cai, on associe des caractéristiques sémantiques et informationnelles différentes. On tente d'expliquer la distribution de jiu dans les principales interrogatives. Pour conclure, on présente quelques caractéristiques distributionnelles des contrefactuelles, en particulier en ce qui concerne le point d'incidence et la portée des négations bu et mei.

Conditional clauses are examined from two points of view: 1) their distribution, 2) their informational status. The semantic polysemy of subordinators is presented as well as the distribution between subordinators and connectors and the cooccurrence restrictions between the subordinators and some head nouns. The subordinate clauses associated with jiu and those associated with cai are opposed in terms of both the semantic and the informational roles they play. A tentative explanation of the distribution of jiu in main clauses is proposed. To conclude some distributional characteristics of counterfactuals - in particular the distribution and scope of the negative markers - are sketched.

\section{AUTEUR}

\section{MARIE-CLAUDE PARIS}

Université Paris 7 D.R.L.

Université Paris 7

2, place Jussieu

75005 - Paris

mcparis@ccr.jussieu.fr 